\title{
Mindfulness-based interventions for substance abuse disorders
}

\begin{abstract}
Substance abuse is a global public health concern. It is associated with various health care problems, increase in crime rates, and decrease in productivity. Despite utilizing various approaches for management of substance abuse worldwide, the substance abuse and relapse rates are still relatively high. This paper introduces a new approach for management of substance abuse disorder utilizing mindfulness-based intervention. This paper presents the positive outcomes associated with using mindfulness for management of substance abuse disorder and suggests utilizing this intervention in various populations.
\end{abstract}

Volume 3 Issue I - 2017

\author{
Ahmad Rayan \\ Psychiatric and mental health nursing, Zarqa University, Jordan \\ Correspondence: Ahmad Rayan, Assistant professor, \\ Psychiatric and mental health nursing, Zarqa University, Jordan, \\ Tel 00962786150103,Email ahmed_rayan87@yahoo.com
}

Received: January 28, 2017 | Published: February 02, 2017

Keywords: mindfulness, intervention, substance abuse, addiction

\section{Opinion}

Recently, addiction is considered a global public health concern. In 2013, about 250 million people addicted harmful substances. Substance abuse and addiction have devastating impacts on the health of individuals worldwide. In 2013, it is estimated that about 187,100 people worldwide died of addiction related consequences. The National Institute on Drug Abuse, in 2015, reported that the consequences of addiction and substance abuse including health care problems, crime, and decrease in productivity at work cost more than 700 billion dollars yearly. ${ }^{2}$

There is a crucial need to find an effective management modality for addiction and substance abuse. Although a variety of interventions are available for people with substance abuse (e.g., CBT and motivational interviewing), the relapse rates are still high, ${ }^{3}$ and addiction on substances and other behaviors is increasing. ${ }^{4}$ Therefore, there is a need to develop an effective intervention to reduce the negative consequences of addiction and substance abuse and prevent relapse worldwide.

Mindfulness-based intervention is considered an innovative and promising approach for management of substance abuse related disorders. ${ }^{5}$ Mindfulness is defined as paying attention and maintaining a non-judgmental acceptance and awareness, moment-by-moment, of the current thoughts, emotions, physical sensations, and the environment. ${ }^{6}$ Mindfulness practice (e.g., mindful breathing) is associated with better physical and psychological health outcomes. ${ }^{7,8}$

Mindfulness practice could manage addictive behaviors and relapse. Mindfulness encourages attention at the current moment which interrupts thought processes associated with substance seeking and provide an innovative coping strategy. ${ }^{9,10}$ It enhances attention toward the current daily life activities and diminishes attention toward substances abused. ${ }^{9}$ Further, mindfulness based interventions teach patients how to cope effectively with stress rather than seeking substances to alleviate stress. ${ }^{9}$

Mindfulness-based interventions could enhance non-judgmental responses to thoughts associated with the use of substances; thereby decrease the risk for relapse. ${ }^{11,12}$ Further, mindfulnessbased interventions could help individuals redirect attention to the body sensations and the current experiences rather than focusing on substances abused. There is a growing interest in examining the effectiveness of different types of mindfulness based interventions on substance abuse and relapse including mindful meditation, Mindfulness-Based Relapse Prevention, Mindfulness-Based Stress Reduction, modified mindfulness training for smoking cessation, Mindfulness-Oriented Recovery Enhancement, and treatments combining mindfulness-based interventions with other interventions. ${ }^{9}$ Furthermore, there are different systematic reviews that supported the usefulness of mindfulness-based interventions to help people with substance abuse. ${ }^{13,14}$ However, the literature suggests that these studies included Western samples with little interest in conducting such studies in Eastern populations. Therefore, there is limited knowledge regarding the effectiveness of mindfulness-based interventions to support patients with substance abuse disorders in Eastern populations. Considering the increased evidences that mindfulness is helpful to support people with substance abuse, there is a need to conduct more studies about the mindfulness-based intervention to manage substance abuse in different populations. Furthermore, there is a need to identify the extent to which treatment effects are maintained after mindfulness-based interventions. Key to this work is how to develop culturally sensitive intervention utilizing mindfulness to be applied in Eastern countries.

\section{Acknowledgements}

None.

\section{Conflict of interest}

The author declares no conflict of interest.

\section{References}

1. United Nations Office of Drugs and Crime. 2015 World drug report finds drug use stable, access to drug \& HIV treatment still low. 2015.

2. National Institute on Drug Abuse. Trends \& statistics. 2015.

3. Tuithof M, ten Have M, van den Brink W, et al. Alcohol consumption and symptoms as predictors for relapse of DSM-5 alcohol use disorder. Drug Alcohol Depend. 2014;140:85-91.

4. 'Rayan A, Dadoul A. M, Jabareen H, et al. Internet Use among University Students in South West Bank: Prevalence, Advantages and Disadvantages, and Association with Psychological Health. International Journal of Mental Health and Addiction. 2016. p. 1-12. 
5. Chiesa A, Serretti A. Are mindfulness-based interventions effective for substance use disorders? A systematic review of the evidence. Subst Use Misuse. 2014;49(5):492-512.

6. Rayan A, Ahmad M. Effectiveness of Mindfulness-Based Intervention on Perceived Stress, Anxiety, and Depression among Parents of Children with Autism Spectrum Disorder. Mindfulness. 2016;7(4):p.1-14.

7. Rayan A, Ahmad M. Mindfulness-based Intervention: A Culturally Adaptable Intervention in Clinical Psychology. Clinical Psychiatry. 2016;2(3):1-2.

8. Rayan A, Ahmad M. Effectiveness of Mindfulness-Based Interventions on Quality of Life and Positive Reappraisal Coping among Parents of Children with Autism Spectrum Disorder. Res Dev Disabil. 2016;55:185196.

9. Garland EL, Froeliger B, Howard, et al. Mindfulness training targets neurocognitive mechanisms of addiction at the attention-appraisalemotion interface. Front Psychiatry. 2013;4:173 p.
10. Rayan A, Ahmad M. Psychological Distress in Jordanian Parents of Children with Autism Spectrum Disorder: The Role of Positive Reappraisal Coping. Arch Psychiatr Nurs. 2017;31(1):38-42.

11. Garland EL, Roberts Lewis A, Tronnier CD, et al. MindfulnessOriented Recovery Enhancement versus CBT for co-occurring substance dependence, traumatic stress, and psychiatric disorders: Proximal outcomes from a pragmatic randomized trial. Behav Res Ther. 2016;77:7-16.

12. Rayan A, Ahmad M. Psychological Distress in Jordanian Parents of Children with Autism Spectrum Disorder: The Role of Trait Mindfulness. Perspect Psychiatr Care. 2016.

13. Katz D, Toner B. A systematic review of gender differences in the effectiveness of mindfulness-based treatments for substance use disorders. Mindfulness. 2013;4(4):318-331.

14.Zgierska A, Rabago D, Chawla N, et al. Mindfulness meditation for substance use disorders: A systematic review. Subst Abus. 2009;30(4):266-294. 